\title{
Modelling Damage and Failure in Adhesive Joints Using A Combined XFEM-Cohesive Element Methodology
}

\author{
A. Mubashar ${ }^{a}$, I. A. Ashcroft ${ }^{b *}$, A. D. Crocombe ${ }^{c}$ \\ ${ }^{a}$ Wolfson School of Mechanical and Manufacturing Engineering, Loughborough University, \\ Loughborough, Leicestershire LE11 3TU, UK \\ ${ }^{b}$ Faculty of Engineering, University of Nottingham, Nottingham \\ 'Division of Mechanical, Medical, and Aerospace Engineering, University of Surrey, Guildford GU2 7HX, \\ UK
}

\begin{abstract}
In recent years, cohesive elements based on the cohesive zone model (CZM) have been increasingly used within finite element analyses of adhesively bonded joints to predict failure. The cohesive element approach has advantages over fracture mechanics methods in that an initial crack doesn't have to be incorporated within the model. It is also capable of modelling crack propagation and representing material damage in a process zone ahead of the crack tip. However, the cohesive element approach requires the placement of special elements along the crack path and is, hence, less suited to situations where the exact crack path is not known a priori. The extended finite element method (XFEM) can be used to represent cracking within a finite element and hence removes the requirement to define crack paths or have an initial crack in the structure. In this paper a hybrid XFEM-cohesive element approach is used to model cracking in the fillet area using XFEM where the crack path is not known and then using cohesive elements to model crack and damage progression along the interface. The approach is applied to the case of an aluminium-epoxy single lap joint and is shown to be highly effective.
\end{abstract}

Keywords: Finite element user models, Epoxy adhesive, Adhesive joint

* Corresponding author. Tel: +44 1509 227535; fax: +44 1509223934

Email address: i.a.ashcroft@lboro.ac.uk(I.A.Ashcroft) 


\section{Introduction}

Structural adhesive joints are being increasingly used in aerospace and automotive industries and reliable methods are needed for predicting joint strength. Analytical solutions for the prediction of adhesive joint strength are limited in their ability to predict damage and failure as they are mostly applicable to simple geometries and may not consider the complex stress states experienced in service environments. Numerical techniques, specifically those based on the finite element method, have provided a means for analysing and predicting the failure of adhesive joints under various loading conditions. The finite element based methods may be classified as strength of materials, fracture mechanics and damage mechanics based methods $[1]$.

The strength of materials methods are based on the study of stresses and strains in an adhesive joint and the application of a suitable stress or strain based failure criterion. Bulk tensile testing of rubber toughened structural adhesives has shown that they exhibit plasticity and elasto-plastic material models may be used to predict their behaviour under load [2-4]. Various failure criteria have been used, in combination with elasto-plastic material models, to predict adhesive joint strength. These include; maximum stress [5, 6], maximum strain $[7,8]$, plastic yielding, maximum principal stress or strain, and plastic energy density [9-15]. Maximum stress and strain based failure criteria were used by Harris and Adams [6] to predict the failure of single lap joints. A non-linear finite element analysis with an elastoplastic material model for the adhesive and adherends was carried out. The selection of stress or strain based failure criterion was based on the results of the uniaxial tensile test results. For an un-toughened adhesive, a brittle failure was observed and a maximum stress criterion was used while for a toughened adhesive, failure was ductile and a maximum strain based criteria was used. However, these criteria are difficult to implement when highly localised stress concentrations or stress singularities exist in an adhesive joint as mesh refinement does not 
provide a converged solution. These methods are also not able to predict crack initiation and growth and can't model any changes in strength as material becomes damaged under loading before failure.

To predict fracture in joints, fracture mechanics methods may be used. Fracture mechanics methods assume that all materials have flaws or cracks and that crack growth will occur when a selected fracture criterion is satisfied. The fracture criterion may be energy based, such as the critical strain energy release rate, $G_{c}$, proposed by Griffith [16], or stress based, such as the stress intensity factor, $K$, given by Irwin [17]. In recent years the J-Integral approach has been used to determine the strain energy release rate through the use of a path independent integral around the crack tip. In linear elastic materials, $\mathbf{J}$ is equal to $\mathrm{G}$, which is strain energy release rate. In nonlinear elastic materials, $\mathrm{J}$ represents the strain energy release rate. The energy based fracture mechanics failure criteria have been used to predict failure in adhesives [18-25]. This criterion dictates that failure will occur when the strain energy release rate equals the critical strain energy release rate. The suitability of strain energy release rate as a failure criterion for adhesive joints was investigated by Hamoush and Ahmad [26] by using compact tension and shear joints to closely resemble mode I and mode II failure. Experimentally obtained failure loads were applied in a finite element model to calculate the strain energy release rates and it was concluded that strain energy release rate was independent of initial crack length and loading mode and thus may be considered a material parameter. Another fracture mechanics based approach for joint strength prediction is based on Irwin's crack closure integral method. It is based on the assumption that the energy absorbed upon crack growth is equal to the work required to close the crack. The method was used by Wahab and De Roeck [27] to calculate strain energy release rate from a threedimensional finite element analysis and good correlation with literature values was reported. Wahab [28] also used the same approach for designing lap joints. One of the limitations of 
the fracture mechanics methods is that they require the existence of a pre-crack to determine the strain energy release rate. Fracture mechanics approaches may require several finite element analyses to determine fracture energies at different crack lengths.

Keeping in view the limitations of strength of materials and fracture mechanics methods, cohesive elements based on the cohesive zone model (CZM) have been used increasingly in recent years to simulate crack initiation, propagation and failure [29-33]. The cohesive elements allow multiple cracks to be modelled and the direction of crack propagation need not be known in advance. The crack can only propagate in a layer of cohesive elements, however, the direction of crack propagation depends on the loading conditions. Cohesive elements are based on traction-separation constitutive law to predict failure initiation, damage and failure. Several shapes for the traction-separation law have been presented in the literature, with the bilinear, linear-parabolic, exponential and trapezoidal shapes being the most commonly used for strength prediction [34-37]. The bilinear law provides a good balance between computational cost and approximation of the problem [38]. The maximum traction in a traction-separation law is known as the tripping traction and may be related to stresses in the normal and shear directions. The parameters input into the cohesive zone model may be determined by a combination of experimental and numerical methods $[39,40]$. Li et al [41] used a cohesive zone model to describe fracture of polymer matrix composite, double cantilever beam (DCB) joints. Fracture was either observed along the interface layer or, in some cases, in the composite adherends. A three parameter cohesive zone model was used to predict failure at the interface and in the composite. Cohesive elements based on cohesive zone models have also been used to investigate the effect of defects on the mechanical behaviour of single lap joints [42]. A two dimensional cohesive element with mixed-mode damage model was employed to predict the failure load of the single lap joints and it was concluded that the maximum load of a joint is decreased only by decrease in 
bonded surface. Blackman et al [43] studied the crack propagation in three types of adhesively bonded joints; DCB, tapered DCB (TDCB) and 90 peel test based on a two parameter CZM approach. The effect of limiting stress parameter was explored in the already mentioned three geometries. For DCB and TDCB specimens, limiting maximum stress affected the predictions of CZM model and the values of the specimen compliance and fracture energy were generally dependent on the limiting stress value until a high value of limiting stress was used. In case of peel test, a lack of unique set of parameters was observed and several pairs of fracture energy and limiting stress were observed to predict the peeling load.

One of the main limitations of the cohesive elements approach is that the cohesive elements should be present on the crack path. Although several layers of cohesive elements may be introduced in a structure, it is not feasible to introduce cohesive elements between every field element, even in a moderate size mesh. The cohesive elements based approach is suitable for crack initiation and propagation for interfacial as well as bulk material. Several methods have been suggested for calibration of the traction-separation laws but a generally accepted method is still required.

A relatively recent enhancement to the finite element method is the extended finite element method (XFEM) [44, 45]. XFEM can be used to predict fracture in a material without prior consideration of the crack path in the finite element mesh. The crack can propagate within elements without adhering to element boundaries and thus alleviates the need to define crack paths or have an initial crack in the structure. The formulation of XFEM is based on the addition of enrichment functions to the finite element nodes. Two types of discontinuities may be present in a mesh; (i) an element is completely separated by crack propagation and (ii) an element contains the crack tip. Thus, enrichment functions are added to the 
displacement field to cater for both types of discontinuities and the displacement vector, $\delta$, takes the form;

$$
\delta=\sum_{k=1}^{S} S_{k}(x)\left[\delta_{k}+H(x) \alpha_{k}+\sum_{i=1}^{4} F_{i}(x) \beta_{k}^{i}\right]
$$

where $S_{k}(x)$ are the finite element nodal shape functions, $H(x)$ is Heaviside function to allow the discontinuity because of the crack growth, $\alpha_{k}$ is a nodal enriched degree of freedom vector, $F_{\alpha}(x)$ is an asymptotic crack tip function and $\beta_{k}^{i}$ is a nodal enriched degree of freedom vector of an element containing a crack tip.

Failure of adhesive joints is generally classified as cohesive or interfacial failure. Cohesive elements may be used for the prediction of interfacial and cohesive crack propagation where CZM elements are present on the expected crack path. XFEM may be used for cohesive crack initiation and growth in adhesive layer without a prior knowledge of crack path. In this paper, a numerical study is carried out to predict the strength of adhesive joints using a combination of XFEM and cohesive elements methods. A single lap adhesive joint was selected for numerical modelling and prediction of joint strength using the proposed hybrid XFEMCohesive element approach. The commercially available finite element code Abaqus was used to build and analyse the finite element models. XFEM and cohesive elements are available as built-in models in Abaqus Standard, which is an implicit finite element code. The results of the numerical modelling were compared with available experimental data for single lap joints. The study revealed various aspects involved during modelling and analysis using the selected methodology. 


\section{Development of Finite Element Model}

A two dimensional single lap joint with aluminium 2024 T3 adherends and FM73-M epoxy adhesive, manufactured by Cytec Engineered Materials Ltd., Wrexham, UK, was modelled in Abaqus (Dassault Systemes, Providence, RI, USA) to predict the residual strength. The single lap joint was selected for this study as it represents a large proportion of joints used in industry. The geometry of the modelled joint is shown in Figure 1. The experimental strength of this type of joint has been reported in [46]. The single lap joint was meshed using quadrilateral four node, incompatible mode elements, which improves the response of the mesh under bending conditions. A continuous mesh, spanning the adhesive layer and the adherends was used. A fine mesh was used in the adhesive layer and element size was increased gradually while moving into the adherends. The mesh size in the adhesive layer was determined after carrying out convergence studies and the adhesive layer was meshed with four elements across the thickness. The element size in the adhesive layer was $0.0248 \mathrm{x}$ $0.025 \mathrm{~mm}$. Half of the single lap joint was modelled by considering the rotational symmetry in the joint geometry and loading as half of the single lap joint may be rotated by $180^{\circ}$ about a point in $2 \mathrm{D}$ space to obtain the complete geometry and loading, as shown in Figure 2 . The joint was loaded under tensile loading conditions up to failure. The mesh and the boundary conditions of the joint finite element model are shown in Figure 2.

An elastic material model was used for the aluminium adherends as no plastic deformation was observed during experimental testing of the joint [46]. The mechanical properties of Al2024 T3 are given in Table 1. The adhesive layer was modelled using a combination of continuum elements with elasto-plastic material model, cohesive elements and XFEM. The elasto-plastic properties of the adhesive were defined based on the experimental stress-strain data obtained by uniaxial tensile testing of bulk adhesive samples as shown in Figure 3 . The cohesive element and XFEM material model parameters are described in next section. 


\section{XFEM-Cohesive Element Methodology}

The methodology used for prediction of single lap adhesive joint strength was based on a combination of XFEM and cohesive element techniques. The methodology was implemented by first creating a finite element model of a single lap joint. The geometry of the finite element model was based on an experimentally tested single lap joint. In the second step, cohesive elements and XFEM material models were defined. Finally, the single lap joint finite element model was loaded under tensile conditions to determine its damage and failure response.

The single lap joint finite element model was embedded with a layer of cohesive elements along the interface between the adherends and the adhesive. A finite thickness of $0.001 \mathrm{~mm}$ was used for the cohesive layer as this thickness is very small compared to the overall dimensions of the joint and adhesive layer and may be considered as negligible. The adhesive layer of the joint was defined as a region of enriched elements where crack can grow in any arbitrary direction, based on XFEM. Thus a combined XFEM-cohesive element model is able to predict crack growth in the adhesive regions close to the interface that would be difficult with a simple XFEM model and is also able to predict a mesh independent crack growth in the adhesive layer and fillet, which is not possible using only a cohesive elements. The cohesive element and XFEM regions are shown in Figure 4.

A bilinear constitutive cohesive zone law was selected for modelling the adhesive region close to the interface. The bilinear cohesive zone law is shown in Figure 5 in the form of a traction-separation response. A stress criterion was used for damage initiation where the damage was assumed to initiate when the ratio of stress at any time increment to the limiting stress reached a value of one, as given by Equation (2). 


$$
\max \left(\frac{\left\langle\sigma_{n}\right\rangle}{\sigma_{n m}}, \frac{\sigma_{s 1}}{\sigma_{s 1 m}}, \frac{\sigma_{s 2}}{\sigma_{s 2 m}}\right)=1
$$

where $\sigma_{n m}$ is the limiting stress in the normal direction, $\sigma_{s 1 m}$ and $\sigma_{s 2 m}$ are the limiting stresses in the two shear directions. $\sigma_{n}, \sigma_{s 1}$ and $\sigma_{s 2}$ are the corresponding stresses at any time increment. " \langle\rangle " is a Macaulay bracket, showing that no damage occurs under pure compression. The tension and shear traction-separation response was considered independent of one another.

The bilinear cohesive zone model may be completely defined by cohesive energy, $G_{c o h}$, stiffness, $K$, and tripping traction, $\tau_{c}$. Turon et al [47] provided a relationship for determining the value of stiffness based on the properties of the surrounding material, which for an isotropic material is given by:

$$
K=\frac{\alpha E}{t}
$$

where $\mathrm{E}$ is the elastic modulus, $t$ is the thickness of surrounding material and $\alpha$ is a parameter whose value should be much larger than $1(\alpha>>1)$. The stiffness of the interface should be large enough that overall compliance is not effected and should be small enough to avoid spurious oscillations in tractions. Thus, the parameter $\alpha$ should provide a reasonable stiffness value and a value of 50 or greater was recommended in [47]. Based on Equation (3), the value of stiffness was calculated to be $8.3 \times 10^{5} \mathrm{~N} / \mathrm{mm}^{3}$, however, convergence difficulties were observed during modelling and a lower value of $1 \times 10^{5}$ was used for the cohesive zone model. The fracture energy of the adhesive FM73, in mixed mode, was determined by Liljedahl et al [48] using a mixed mode flexure (MMF) specimen and a value of $2.9 \mathrm{KJ} / \mathrm{m}^{2}$ was reported. 
A penalty based cohesive zone finite element approach, as suggested by Diehl $[49,50]$ was used to determine $\tau_{c}$. The approach behind the penalty method is to use strain energy release rate as the main parameter in a CZM material model and convert the CZM law into a rigid assumption, as is used in the classical Griffith criterion. The classical methods of fracture mechanics assume that material behaviour is infinitely stiff in the bonding direction until failure occurs. A 2D single lap joint finite element model with a layer of cohesive elements, which were embedded in the mesh based on the experimental observations, was used to determine $\tau_{c}$. Two dimensional, continuum four node, plane strain elements were used for the adhesive and the adherends. The single lap joint was loaded in displacement control up to failure and the finite element predicted failure load was calibrated to the experimental failure load, which gave a value of $39 \mathrm{MPa}$ for $\tau_{c}$. The finalised parameters for the bilinear cohesive zone law are given in Table 2.

The damage and failure behaviour of the XFEM model was based on a maximum principal stress criterion and follows a linear damage curve as shown in Figure 6. Damage initiation occurs when a maximum principal stress values is reached at any point in the material. The damage propagation in the XFEM elements is based on a scalar damage variable, $D$, whose value increases from 0 to 1 where 0 represents no damage in the material and 1 represents fully damaged material. The damage evolution follows a linear law and failure occurs when the ratio of maximum principal stress $\left(\sigma_{\text {prin }}\right)$ to maximum allowable principal stress ( $\left.\sigma_{\text {prin_max }}\right)$ reaches a value of one. The maximum principal stress damage initiation and propagation criterion is given by;

$$
\frac{\left\langle\sigma_{p r i n}\right\rangle}{\sigma_{p r i n \_\max }}
$$


The stresses are determined based on the damage variable as follows;

$$
\begin{aligned}
& \sigma_{t}=(1-D) \sigma_{T}, \sigma_{T} \geq 0 \text { else } \sigma_{T} \\
& \sigma_{s 1}=(1-D) \sigma_{S 1} \\
& \sigma_{s 2}=(1-D) \sigma_{S 2}
\end{aligned}
$$

where $\sigma_{T}, \sigma_{S 1}$ and $\sigma_{S 2}$ are the elastic components of stresses before damage. The XFEM governing law is completely defined by defining the maximum principal stress at initiation and displacement at failure. A value of $54 \mathrm{MPa}$ was used for maximum principal stress. The maximum tensile stress from the uniaxial tensile stress strain curve was used for the maximum principal stress and displacement at failure was taken as the displacement measured between the maximum tensile stress and rupture. The cohesive energy used for XFEM was the same as that used for the cohesive elements, following the logic that apparent interfacial failure in a bonded joint with a good surface treatment is often actually in the adhesive material adjacent to the interface. This was the case in the experiments used to validate the proposed model, as described in the next section, however, in the case of a 'true' interfacial fracture a different cohesive energy should be used.

\section{Results and Discussion}

The force displacement response of the single lap joint under tensile loading is shown in Figure 7. The failure force predicted by the finite element model is within the limits of the experimentally observed failure force. The predicted force displacement response remains linear up to $10.8 \mathrm{kN}$, after which a nonlinear force displacement response was observed. The nonlinear behaviour observed is owing to evolving damage in the adhesive. This results in decreased load carrying capacity of the adhesive which would not be predicted if a material 
model without damage mechanics was used. A sudden failure occurs as the failure load is reached and the adhesive is no longer able to sustain the applied load.

The failure path in the joint is shown in Figure 8 where crack propagation in both XFEM and cohesive elements can be seen. The crack in the adhesive fillet region occurs in the XFEM domain and can be seen to propagate through the elements without being constrained by the element boundaries. Failure initiation occurred in the overlap end region, near the lower adherend corner, as shown in Figure 9a. The interface of the adherend and the adhesive produces a numerical singularity owing to the sharp adherend corner. A finer mesh is already in use in this region because of the small thickness of the adhesive layer. Also further decrease in element size in this region did not affect the overall failure behaviour of the joint. The crack then extends towards the upper adherend as the load is increased and passes through the adhesive layer as may be seen in Figure 9b. As the crack reaches the cohesive elements, crack development in the XFEM domain stops and cohesive elements start to show damage. The failure of cohesive elements may be seen in Figure 9c. Meanwhile, crack propagation in the XFEM domain continues through the adhesive fillet. The simultaneous development of the crack continues in the XFEM and cohesive zone layer and the crack in the fillet reaches the fillet boundary. On the other hand, the crack in the cohesive elements extends towards the middle of the joint. Since, half of the joint is modelled, a similar initiation and growth of crack in the remaining half of the joint is assumed.

The experimental results of failure of such an adhesive joint are shown in Figure 10 and were published in [46]. For unconditioned joints, a cohesive failure was observed in the experimental tests. Failure in the fillet occurred in the middle of the fillet, as was observed during the modelling of the joint. The crack grew in the adhesive layer, close to the interface, in the experimental joints. The cohesive element layer was embedded close to the interface in the modelled joint. The direction of crack growth in both experimental and modelled 
adhesive joint was similar as the crack initiated in the fillet and grew towards the middle of the joint.

Based on the present limitations of the XFEM implementation in the Abaqus finite element code, a few observations in crack initiation and propagation may be observed. The initiation of a crack in the XFEM domain has to start in the middle of an element and thus crack initiation in the single lap joint starts in middle of the element close to the singularity region. The crack is also not able to propagate at the interface of different material regions and thus may be arrested if such a condition occurs. The interaction of cohesive zone and XFEM domain cracks is shown in Figure 11. The XFEM crack ends in the middle of the adhesive element and cohesive elements start to observe damage before failing under load. Since one complete cohesive element has to fail, the XFEM and cohesive element cracks are not connected at their ends and the cohesive zone crack extends the XFEM crack by the element length. However, since the element length is small, this does not induce a major effect on the overall load bearing capacity of the joint. The crack propagation in XFEM occurs orthogonal to the maximum principal stress / strain and thus mesh size dependence may affect the damage initiation [45]. If XFEM was used for crack propagation in the adhesive layer as well as in the fillet, the crack initiating in the fillet would grow towards the adherend based on the principal stress / strain direction. Since the crack cannot continue in the adherend, it would continue to grow at the interface [44]. This would not be representative of the experimental failure in the single lap adhesive joints where cohesive failure occurs in the adhesive layer. By using the suggested method of combining the cohesive elements and XFEM, a layer of cohesive elements in the adhesive layer may be used to simulate cohesive failure at the experimentally observed site of failure. 


\section{Conclusions}

XFEM has been shown to be able to accurately predict the crack path in the fillet region of the adhesive in an adhesive joint. This is achieved without needing to align the element edges to the crack path as the XFEM method enables cracking to be represented within elements.

The XFEM can be combined with the CZM approach to effectively predict crack initiation and growth in the adhesive fillet region leading to damage and crack growth in the interfacial region between the adhesive and adherend.

There are some limitations to the approach, in particular, the current implementation of XFEM and CZM means that there is a potential discontinuity in the crack when it moves from the XFEM to CZM domain. However, this is not a major drawback compared with the advantages of being able to efficiently and accurately predict failure imitation and propagation without pre-defining the failure path. 


\section{References}

[1] Da Silva, L.F.M. and Campilho, R.D.S.G., Advances in Numerical Modelling of Adhesive Joints. (Springer, 2012).

[2] Cui, J., Wang, R., Sinclair, A.N., and Spelt, J.K., Int. J. Adhesion and Adhesives. 23, 199-206 (2003).

[3] Feih, S. and Shercliff, H.R., Int. J. Adhesion and Adhesives. 25, 47-59 (2005).

[4] Dean, G., Crocker, L., Read, B., and Wright, L., Int. J. Adhesion and Adhesives. 24, 295306 (2004).

[5] Broughton, W.R., Crocker, L.E., and Urquhart, J.M., Strength of adhesive joints: A parametric study. 2001, National Physical Laboratory (NPL).

[6] Harris, J.A. and Adams, R.D., Int. J. Adhesion and Adhesives. 4, 65-78 (1984).

[7] Hart-Smith, L.J., Adhesive bonded single lap joints. 1973, NASA.

[8] Hart-Smith, L.J., Analysis and design of advanced composite bonded joints. 1974, NASA.

[9] Adams, R.D., Atkins, R.W., Harris, J.A., and Kinloch, A.J., J. Adhesion. 20, 29-53 (1986).

[10] Adams, R.D., J. Adhesion. 30, 219-242 (1989).

[11] Lee, S.J. and Lee, D.G., J. Adhesion. 40, 1-14 (1992).

[12] Hollaway, L., Romhi, A., and Gunn, M., Composite Structures. 16, 125-170 (1990).

[13] Adams, R.D. and Harris, J.A., Int. J. Adhesion and Adhesives. 7, 69-80 (1987).

[14] Zhao, X., Adams, R.D., and da Silva, L.F.M., Journal of Adhesion Science and Technology. 25, 837-856 (2011).

[15] Chen, Z., Adams, R.D., and da Silva, L.F.M., Engineering Fracture Mechanics. 78, 990-1007 (2011).

[16] Griffith, A.A., Philosophical Transactions of the Royal Society. A221, 163-198 (1920).

[17] Irwin, G.R., J Appl Mech. 24, 361-364 (1957).

[18] Ashcroft, I.A., Hughes, D.J., and Shaw, S.J., Int. J. Adhesion and Adhesives. 21, 87-99 (2001).

[19] Imanaka, M., Nakamura, Y., Nishimura, A., and Iida, T., Composites Science and Technology. 63, 41-51 (2003).

[20] Chai, H. and Chiang, M.Y.M., Journal of the Mechanics and Physics of Solids. 44, 1669-1689 (1996).

[21] Chai, H. and Chiang, M.Y.M., Int. J. Solids and Structures. 35, 815-819 (1998).

[22] Daghyani, H.R., Ye, L., and Mai, Y.W., J. Mat. Sci. 31, 2523-2529 (1996).

[23] Ashcroft, I.A., J. Strain Analysis. 39, 707-716 (2004).

[24] Karachalios, E.F., Adams, R.D., and da Silva, L.F.M., Int. J. Adhesion and Adhesives. 43, 96-108 (2013).

[25] Chen, Z., Adams, R.D., and da Silva, L.F.M., Int. J. Adhesion and Adhesives. 31, 48-55 (2011). 
[26] Hamoush, S.A. and Ahmad, S.H., Int. J. Adhesion and Adhesives. 9, 171-178 (1989).

[27] Wahab, M.M.A. and de Roeck, G., Engineering Fracture Mechanics. 49, 659-665 (1994).

[28] Wahab, M.A., Journal of Adhesion Science and Technology. 14, 851-865 (2000).

[29] Zhang, L. and Wang, J., Int. J. Adhesion and Adhesives. 29, 217-224 (2009).

[30] Ural, A., Krishnan, V.R., and Papoulia, K.D., Int. J. Solids Struct. 46, 2453-2462 (2009).

[31] Zavattieri, P.D., Hector Jr, L.G., and Bower, A.F., Engineering Fracture Mechanics. 75, 4309-4332 (2008).

[32] Freed, Y. and Banks-Sills, L., Engineering Fracture Mechanics. 75, 4583-4593 (2008).

[33] Carlberger, T. and Stigh, U., Engineering Fracture Mechanics. 74, 2247-2262 (2007).

[34] Sun, C., Thouless, M.D., Waas, A.M., Schroeder, J.A., and Zavattieri, P.D., Int. J. Solids Struct. 45, 4725-4738 (2008).

[35] Meo, M. and Thieulot, E., Composite Structures. 71, 429-434 (2005).

[36] Fan, C., Jar, P.Y.B., and Cheng, J.J.R., Engineering Fracture Mechanics. 75, 38663880 (2008).

[37] Chandra, N., Li, H., Shet, C., and Ghonem, H., Int. J. Solids Struct. 39, 2827-2855 (2002).

[38] Alfano, G., Composites Science and Technology. 66, 723-730 (2006).

[39] Gustafson, P.A. and Waas, A.M., Int. J. Solids Struct. 46, 2201-2215 (2009).

[40] Goyal, V.K., Johnson, E.R., and Goyal, V.K., Composite Structures. 82, 434-446 (2008).

[41] Li, S., Thouless, M.D., Waas, A.M., Schroeder, J.A., and Zavattieri, P.D., Composites Science and Technology. 65, 281-293 (2005).

[42] de Moura, M.F.S.F., Daniaud, R., and Magalhães, A.G., Int. J. Adhesion and Adhesives. 26, 464-473 (2006).

[43] Blackman, B.R.K., Hadavinia, H., Kinloch, A.J., and Williams, J.G., International Journal of Fracture. 119, 25-46 (2003).

[44] Campilho, R.D.S.G., Banea, M.D., Pinto, A.M.G., da Silva, L.F.M., and de Jesus, A.M.P., Int. J. Adhesion and Adhesives. 31, 363-372 (2011).

[45] Campilho, R.D.S.G., Banea, M.D., Chaves, F.J.P., and Silva, L.F.M.d., Computational Materials Science. 50, 1543-1549 (2011).

[46] Mubashar, A., Ashcroft, I.A., Critchlow, G.W., and Crocombe, A.D., Int. J. Adhesion and Adhesives. 29, 751-760 (2009).

[47] Turon, A., Davila, C.G., Camanho, P.P., and Costa, J., Engineering Fracture Mechanics. 74, 1665-1682 (2007).

[48] Liljedahl, C.D.M., Crocombe, A.D., Wahab, M.A., and Ashcroft, I.A., Int. J. Adhesion and Adhesives. 27, 505-518 (2007).

[49] Diehl, T., Int. J. Adhesion and Adhesives. 28, 237-255 (2008).

[50] Diehl, T., Int. J. Adhesion and Adhesives. 28, 256-265 (2008). 
[51] Kutz, M., ed. Handbook of Materials Selection. 2002, John Wiley \& Sons, Inc.: New York. 


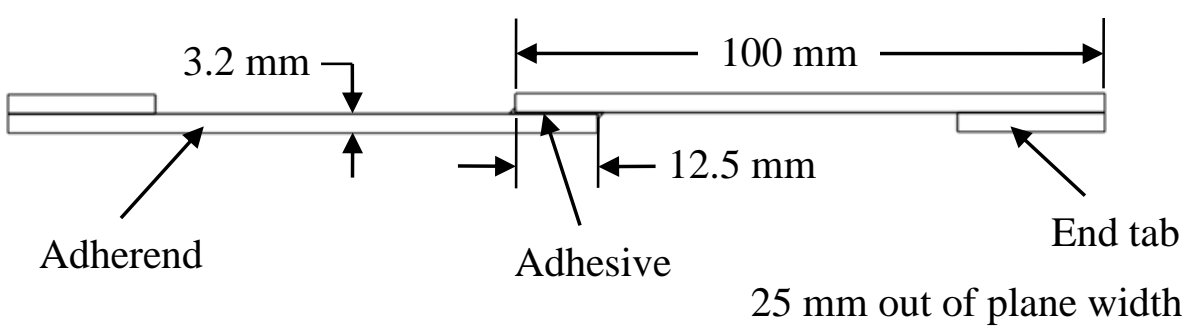

Figure 1: Single lap joint configuration and geometry.

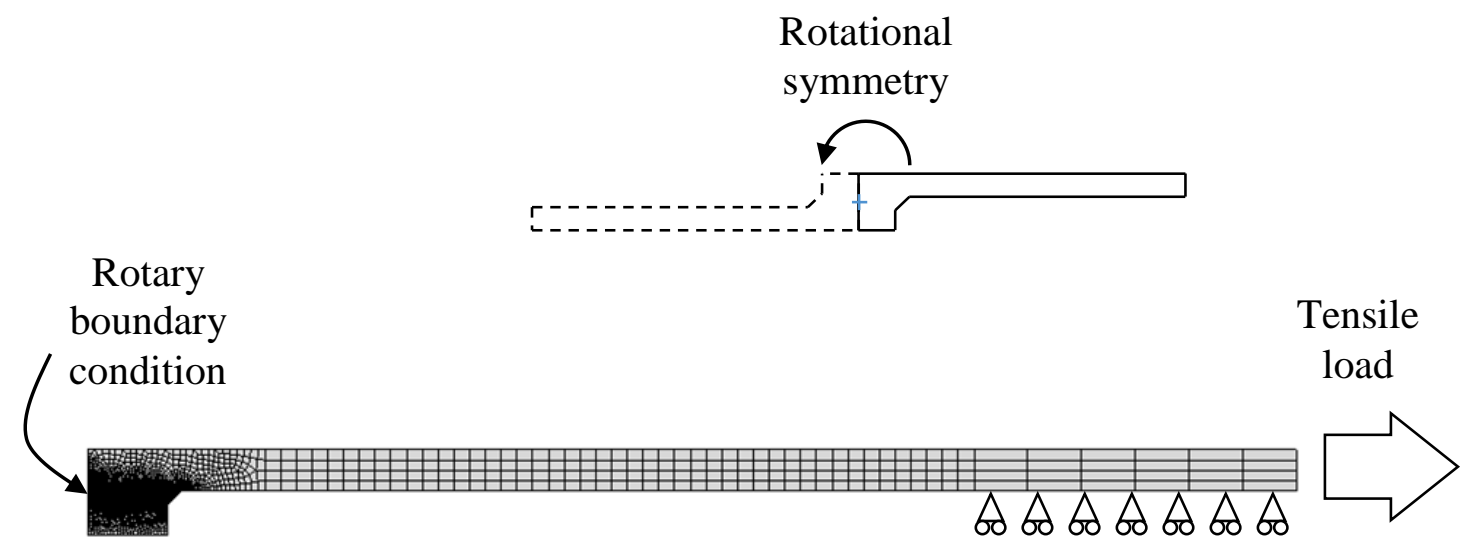

Figure 2: Mesh and boundary conditions of the finite element model of single lap joint. 


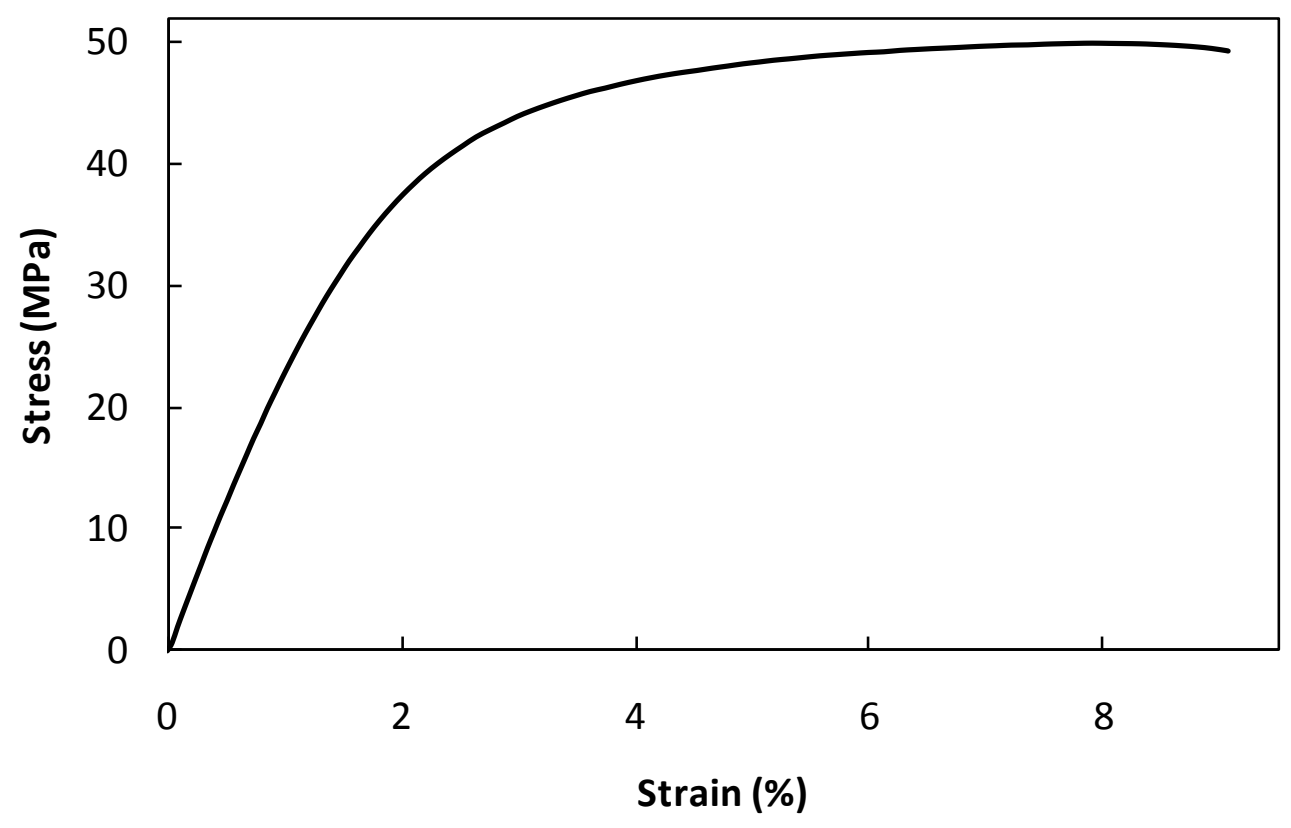

Figure 3: Experimental tensile stress strain (engineering) behaviour of bulk adhesive samples.

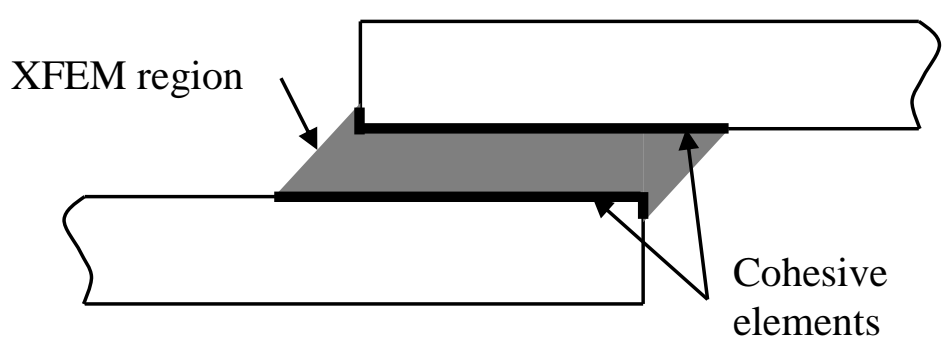

Figure 4: Regions of cohesive zone and XFEM elements. 


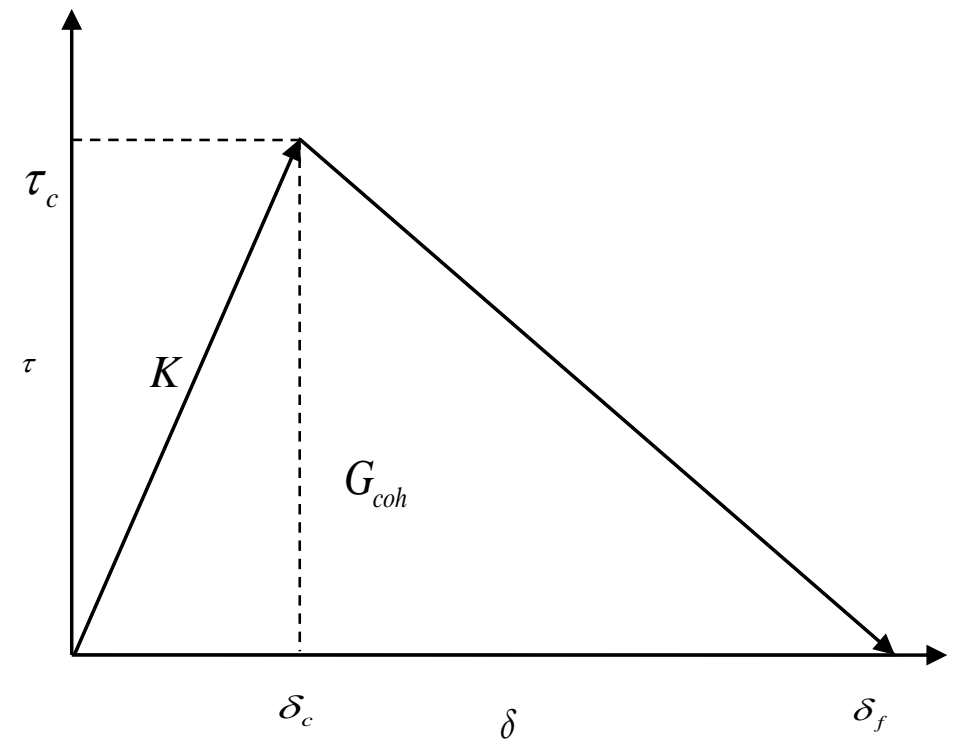

Figure 5: Bilinear cohesive zone law.

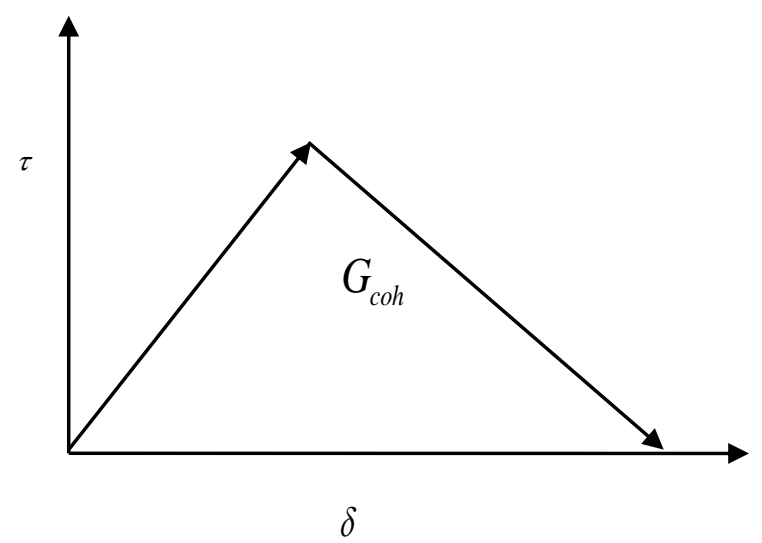

Figure 6: Linear traction-separation law governing the XFEM material model. 


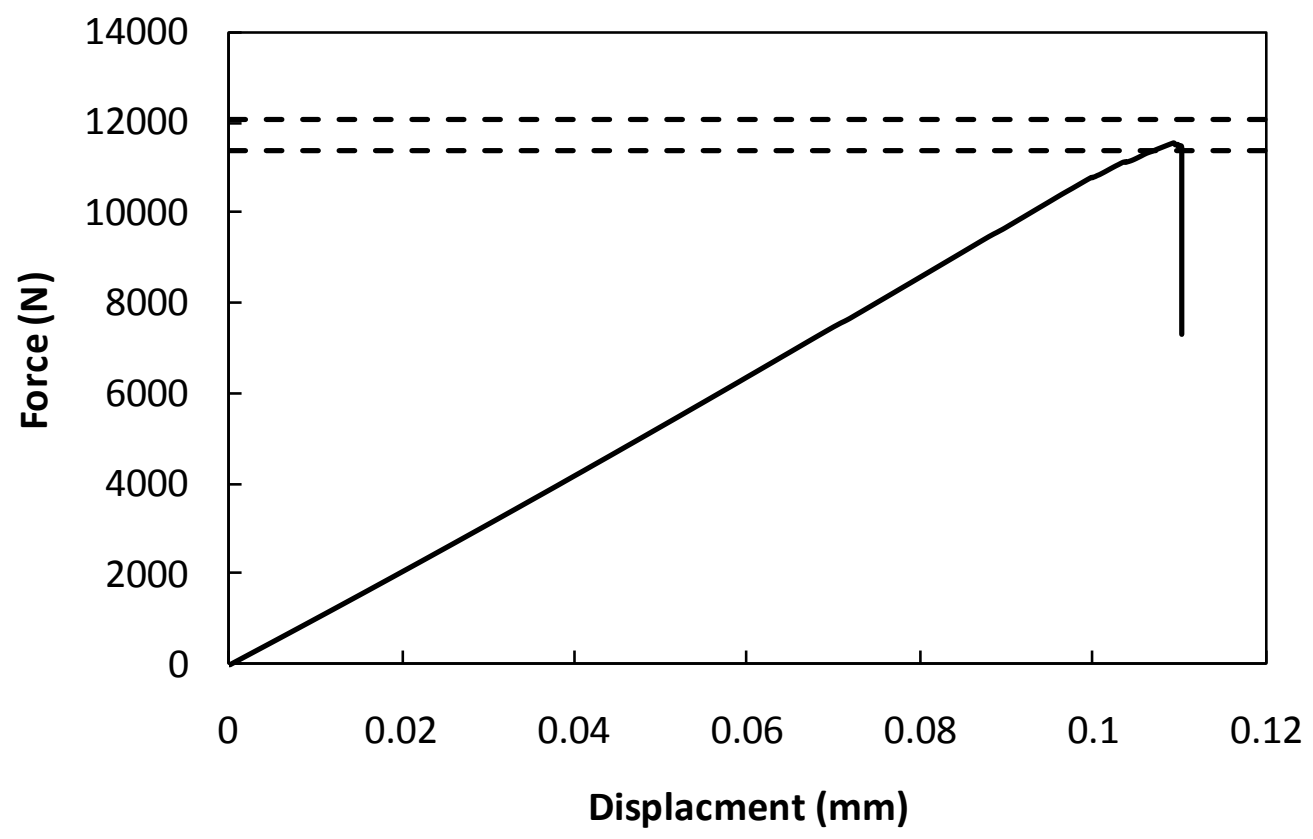

Figure 7: Force-displacement response of the XFEM-CZM based single lap joint. The dotted lines show the range of experimental results.

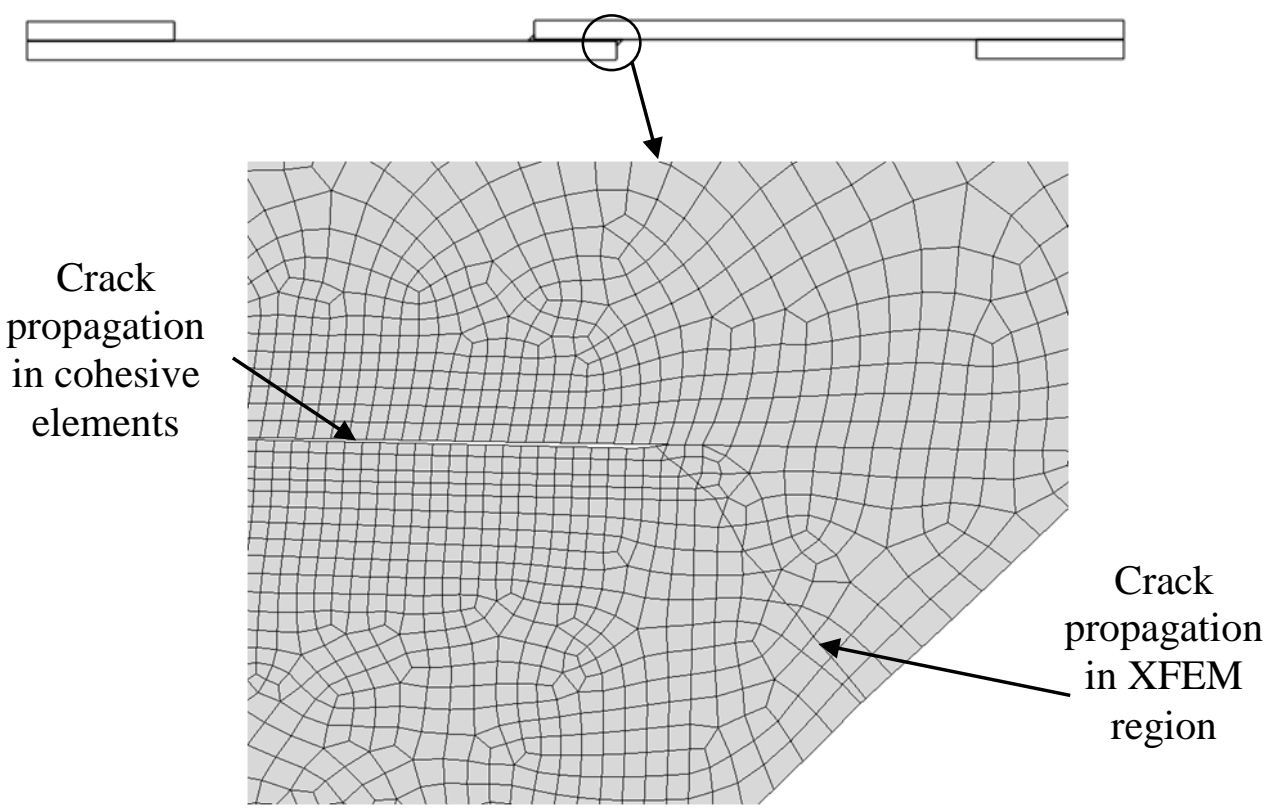

Figure 8: Crack propagation in the finite element single lap joint model. 


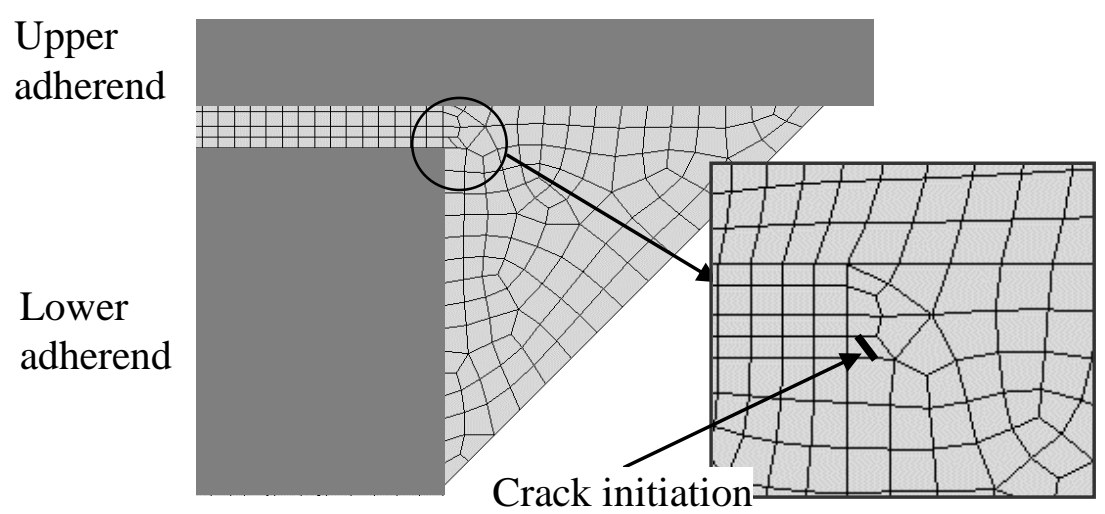

(a)

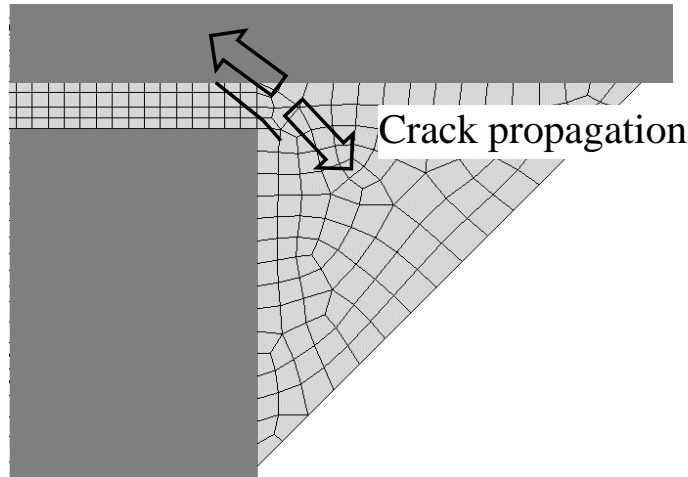

Crack propagation in cohesive layer

(b)

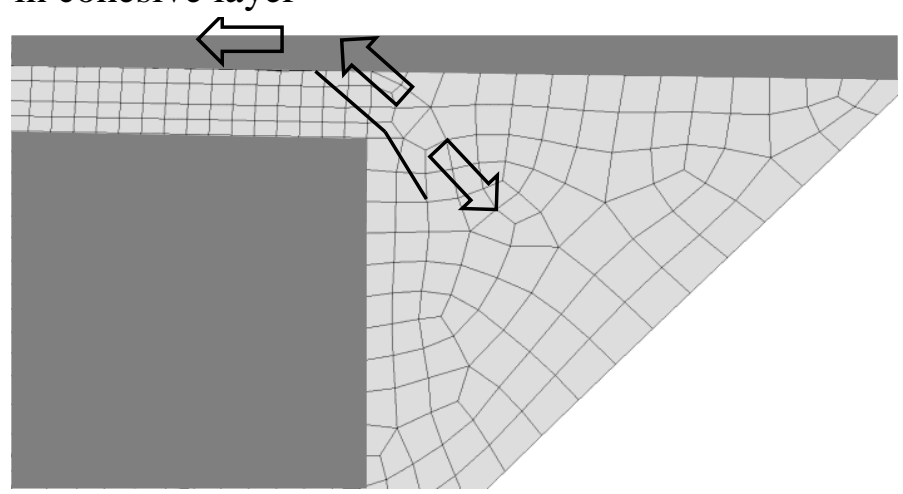

(c)

Figure 9: Crack initiation and development in the adhesive layer. 

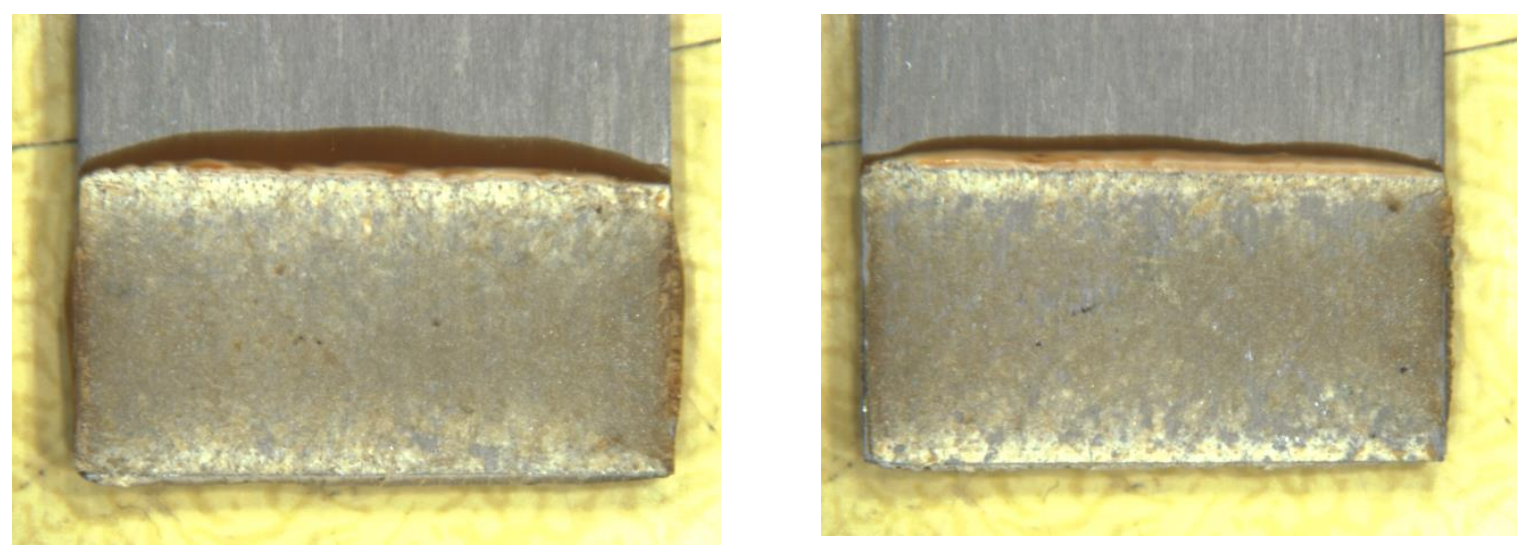

Figure 10: Experimental failure surfaces of an aluminium 2024 single lap joint.

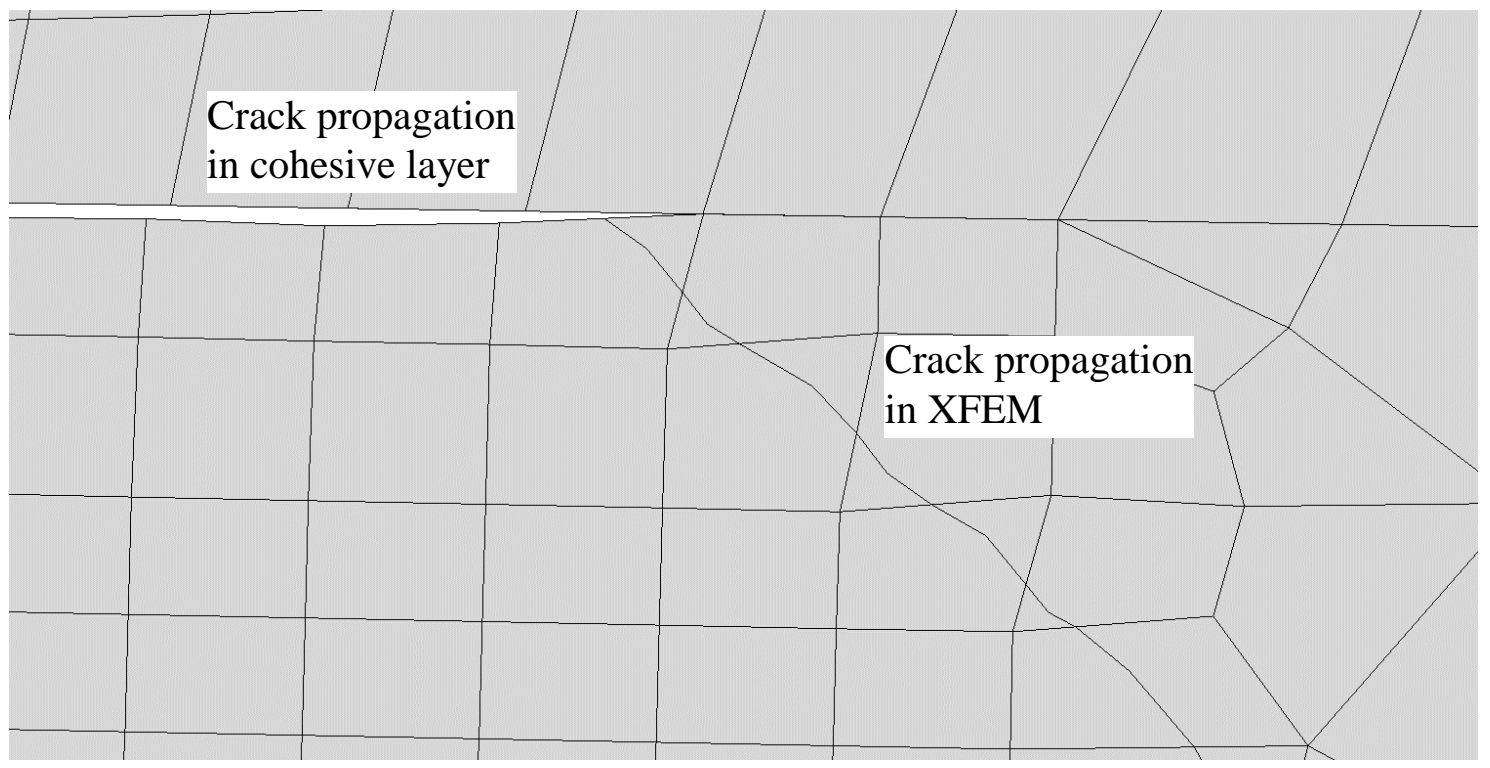

Figure 11: Interaction of XFEM and cohesive zone domain cracks. 
Table 1: Mechanical properties of aluminium alloy 2024 T3 [51].

\begin{tabular}{lc}
\hline \multicolumn{1}{c}{ Mechanical Property } & Value \\
\hline Elastic modulus (GPa) & 73 \\
Poisson's ratio & 0.33 \\
Yield Strength (MPa) & 345 \\
\hline
\end{tabular}

Table 2: The cohesive zone model parameters for single lap joint.

\begin{tabular}{ccc}
\hline $\begin{array}{c}\text { Tripping traction } \\
\tau_{c}(\mathrm{MPa})\end{array}$ & $\begin{array}{c}\text { Stiffness } \\
K(\mathbf{N} / \mathbf{m m})\end{array}$ & $\begin{array}{c}\text { Cohesive energy } \\
G_{c o h}\left(\mathbf{k J} / \mathbf{m}^{2}\right)\end{array}$ \\
\hline 39 & $1 \times 10^{5}$ & 2.9 \\
\hline
\end{tabular}

